\title{
Tropospheric corrections to SAR interferometry from GPS observations
}

\author{
Volker Janssen, Linlin Ge and Chris Rizos \\ School of Surveying and Spatial Information Systems \\ The University of New South Wales \\ Sydney NSW 2052, Australia
}

\begin{abstract}
Interferometric Synthetic Aperture Radar (InSAR) techniques have been recognised as an ideal tool for many ground deformation monitoring applications. However, the spatially and temporally variable delay of the radar signal propagating through the atmosphere is a major limitation to accuracy. The dominant factor to be considered is the tropospheric heterogeneity, which can lead to misinterpretation of InSAR results. In this paper a between-site and between-epoch double-differencing algorithm for the generation of tropospheric corrections to InSAR results based on GPS observations is tested. In order to correct the radar results on a pixel-by-pixel basis, the GPS-derived corrections have to be interpolated. Using experimental data it has been found that the inverse distance weighted and kriging interpolation methods are more suitable than the spline interpolation method. Differential corrections as large as several centimetres may have to be applied in order to ensure subcentimetre accuracy for the InSAR result. The algorithm and procedures described in this paper could easily be implemented in a continuous GPS network data centre. The interpolated image of between-site, singledifferenced tropospheric delays can be derived as a routine product to assist radar interferometry.
\end{abstract}

\section{Introduction}

Interferometric Synthetic Aperture Radar (InSAR) is a technique first suggested in the early 1970s (Graham 1974). The technique produces an 'interferogram' from the phase difference between two SAR images acquired over the same region. The satellites which currently acquire SAR images are ERS-2 and Radarsat-1. Envisat was launched in March 2002, and new radar satellites are planned for launch over the next few years, e.g. the Japanese Advanced Land Observation Satellite (ALOS).

The InSAR interferogram contains several types of information: 1) topographic pattern, a contour-like pattern representing the topography of the area; 2) geometric pattern, a systematic striped pattern caused by differences between the two SAR sensor trajectories; and 3) differential pattern, fringes associated with any change of the range between the two SAR images, the sources of which include ground displacement, change of atmospheric refraction, and phase change by reflection due to, for example, growth of vegetation. The geometric pattern can be removed by modelling the geometry of the satellite orbits and ground targets. Unless a highresolution digital elevation model (DEM) is available, an additional radar image is necessary to remove the topographic pattern from the interferogram. In a typical ERS three-pass InSAR procedure, two repeat-pass ERS2 images will be processed to generate the interferogram (InSAR result 1), containing all the information mentioned earlier. A third ERS-1 image which forms a tandem pair with one of the two ERS-2 images is also introduced. The tandem pair with the ERS-2 satellite following the ERS-1 satellite one day later can be processed to generate the topographic pattern (InSAR result 2), because the deformation and growth of vegetation, etc., within one day can be neglected. By differencing the two InSAR results, the residual interferogram will contain only the differential pattern.

Due to its high spatial resolution, ability of SAR to penetrate clouds, and cost effectiveness, InSAR has definite advantages over many conventional deformation monitoring techniques. Many earthquake rupture zones and volcanoes have been studied using InSAR (Massonnet et al. 1993, Lu et al. 1997). Studies, however, have shown that a change of atmospheric refraction (e.g. caused by a cold front moving across the region being imaged) can result in biases, which can lead to a misinterpretation of the InSAR results (Zebker et al. 1997, Hanssen et al. 1999). Therefore, in order to reliably derive ground displacement from InSAR results, it is crucial to correct for the atmospheric heterogeneity.

The atmospheric heterogeneity can be partitioned into tropospheric and ionospheric portions. In general, the troposphere can be divided into a wet component and a dry component. The ionosphere extends, in a number of distinct layers, from about $50 \mathrm{~km}$ to $1000 \mathrm{~km}$ above the Earth's surface. The SAR satellite orbit altitudes are typically in the range of $600-800 \mathrm{~km}$. The effect of the variations caused by the ionospheric layers lower than the SAR satellite altitude will be much smaller than that from the troposphere because the area penetrated by the radar is much smaller. For example, for a SAR satellite at $800 \mathrm{~km}$ altitude the extend of the image in the ionosphere at the height of $400 \mathrm{~km}$ is $40 \mathrm{~km}$, while it will be about $80 \mathrm{~km}$ within the troposphere. Therefore, the ionospheric delay on the radar signal is usually considered to be uniform within one SAR image and can mostly cancel because the SAR images are acquired at the same time of the day, and hence the residual effect can be neglected. It is the tropospheric variations that can lead to misinterpretation of InSAR results. While the dry component of the tropospheric delay is well modelled, the wet component is much more difficult to model 
because of the large variations of water vapour content with respect to time and space (Spilker 1996).

Since 1997 researchers have been developing methodologies to correct InSAR results for these biases using measurements from other techniques, such as GPS (e.g. Bock and Williams 1997, Ge et al. 1997, Ge 2000). However, progress has been slow because in order to integrate InSAR with GPS both datasets have to be available for the same region, at the same time, and the region under study has to be experiencing ground displacement. The establishment of continuous GPS (CGPS) arrays in many parts of the world has eased such difficulties significantly (SCIGN 2003, GSI 2003).

In this paper a between-site and between-epoch double-differencing algorithm for the generation of tropospheric corrections to InSAR results based on GPS observations, as proposed by Hanssen (2001), is tested. The tropospheric parameters are interpolated in order to enable the radar results to be corrected on a pixel-bypixel basis. Experimental results generated from data collected in two CGPS networks are presented.

\section{GPS-derived tropospheric delay}

The troposphere can be defined as the neutral (i.e. non-ionized) part of the atmosphere that stretches from the Earth's surface to a height of approximately $50 \mathrm{~km}$. The dominant impact of tropospheric path delay on radio signals occurs in the lower part, typically below 10km (Spilker 1996). The tropospheric delay is dependent on temperature, atmospheric pressure and water vapour content. The type of terrain below the signal path can also have an effect. The tropospheric effect can be divided into two components, the dry and the wet component. The dry component accounts for about $90 \%$ of the effect and can be accurately modelled using surface measurements of temperature and pressure. However, due to the high variation in the water vapour content, it is very difficult to model the remaining wet component.

Several models based on a 'standard atmosphere' have been developed to account for the tropospheric delay in the absence of accurate ground meteorological data, e.g. the Hopfield model (Hopfield 1969), Saastamoinen model (Saastamoinen 1973) and Black model (Black 1978). As recommended by Mendes (1999), the Saastamoinen model has been used in this study. This model utilizes the gas laws to deduce refractivity, and the tropospheric delay is therefore a function of zenith angle, pressure, temperature and the partial pressure of water vapour. Saastamoinen (1973) used the refractivity constant given by Essen and Froome (1951) for mid-latitudes and average conditions. The original model has subsequently been refined to include two correction terms: one being dependent on the station height $(B)$ and the other on the height and the zenith angle $(\delta R)$. Both terms can be obtained from tables.

The tropospheric delay, expressed in metres, is then given by Bauersima (1983):

$$
d \text { Trop }_{\text {apr }}=\frac{0.002277}{\cos z}\left[p+\left(\frac{1255}{T}+0.05\right) e-B \tan ^{2} z\right]+\delta R
$$

where $z$ denotes the zenith angle of the satellite, $p$ the atmospheric pressure in millibars, $T$ the temperature in Kelvin, and $e$ the partial pressure of water vapour in millibars.

For high precision surveys, an additional parameter can be introduced into the least squares reduction of the observations to estimate the residual tropospheric delay (after modelling). The total tropospheric delay correction $d \operatorname{Trop}_{k}^{i}$ can be expressed as (Rothacher and Mervart 1996):

$$
d \operatorname{Trop}_{k}^{i}=d \operatorname{Trop}_{a p r, k} f_{\text {apr }}\left(z_{k}^{i}\right)+d \operatorname{Trop}_{k}(t) f\left(z_{k}^{i}\right)
$$

where $d T_{r o p} p_{a p r, k}$ denotes the tropospheric delay according to the a priori model, which is time-invariant (i.e. dependent on the station height only) if a standard atmosphere is used. $z_{k}^{i}$ denotes the zenith angle (for satellite $\mathrm{i}$ and station k), $f_{a p r}$ the mapping function (different for each a priori model), $d \operatorname{Trop}_{k}(t)$ the time-dependent troposphere parameter for station $\mathrm{k}$, and $f\left(z_{k}^{i}\right)$ is the mapping function used for the parameter estimation, which may be different from $f_{a p r}$ and is usually $1 / \cos z$.

In this study the Bernese GPS processing software was used to derive tropospheric delay parameters for the individual stations of the network during parameter estimation. The user can specify the number of correction parameters to be estimated within the observation period.

\section{Double-differencing algorithm for tropospheric delay corrections}

Only the relative tropospheric delay (the tropospheric heterogeneity) between two SAR imaging points and between the two SAR image acquisitions will distort the deformation information derived by InSAR, because it is the phase difference that is used and deformation is always referenced to a stable point (site) in the image. Therefore, a between-site and between-epoch double-differencing algorithm can be used to derive the corrections to the InSAR result from GPS observations (Hanssen 2001). 


\subsection{Single-differences}

Assume that A is a stable site in the SAR image to be used as a reference point. B is another site in the same SAR image. If the tropospheric delay estimated from GPS for A and B at SAR imaging epoch $\mathrm{j}$ is denoted as $D_{A}^{j}$ and $D_{B}^{j}$ respectively, the between-site difference of the delays is:

$$
D_{A B}^{j}=D_{B}^{j}-D_{A}^{j}
$$

Using site A as the reference, single between-site difference delays at other GPS sites can also be calculated using equation (3), which are then interpolated (see next section) to generate a tropospheric delay image product similar to the radar SLC (single-look-complex) data.

\subsection{Double-differences}

Assuming two sites A and B, and two epochs $\mathrm{j}$ (master SLC image) and $\mathrm{k}$ (slave image), two singledifferences may be formed according to equation (3):

$$
\begin{aligned}
& D_{A B}^{j}=D_{B}^{j}-D_{A}^{j} \\
& D_{A B}^{k}=D_{B}^{k}-D_{A}^{k}
\end{aligned}
$$

A double-difference is obtained by differencing these single-differences:

$$
\begin{aligned}
D_{A B}^{j k} & =D_{A B}^{k}-D_{A B}^{j} \\
& =\left(D_{B}^{k}-D_{A}^{k}\right)-\left(D_{B}^{j}-D_{A}^{j}\right) \\
& =\left(D_{B}^{k}-D_{B}^{j}\right)-\left(D_{A}^{k}-D_{A}^{j}\right)
\end{aligned}
$$

Equation (5) illustrates two possible approaches to double-differencing, either between-site (BS) differencing first and then between-epoch (BE) differencing (BSBE approach), or between-epoch differencing first and then between-site differencing (BEBS approach). The BSBE approach is preferred because the BS difference can be interpolated to generate a single-difference correction product. This product will be associated with only the SLC image and hence can be used freely to form combinations for further BE differences as soon as InSAR pairs have been formed from SLC images.

\section{Interpolating tropospheric delay corrections}

Continuous GPS networks may be as dense as one station every $25 \mathrm{~km}$ at the national level, as is the case for the GEONET in Japan (GSI 2003), or as dense as one station every few kilometres at the regional level, as is the case for the SCIGN in the USA (SCIGN 2003). However, in order to correct the InSAR result on a pixel-bypixel basis (ERS SAR resolution $\sim 25 \mathrm{~m}$ ), the GPS-derived tropospheric corrections have to be interpolated.

In this section the utility of three interpolating methods will be discussed. Each interpolation technique makes assumptions about how to determine the estimated (interpolated) values. Depending on the phenomenon being modelled (i.e. differential tropospheric delay) and the distribution of sample points (in this case, GPS stations), one interpolator may produce better models of the actual surface (the tropospheric delay correction model) than others. Regardless of the interpolator, as a rule-of-thumb, the more input points and the more even their distribution, the more reliable the results.

\subsection{Inverse distance weighted (IDW) interpolation}

Inverse distance weighted interpolation (Lancaster and Salkauskas 1986) explicitly assumes that things that are close to one another are more alike than those that are farther apart. To predict a value for any unmeasured location, IDW will use the measured values surrounding the prediction location. Those measured values closest to the prediction location will have more influence on the predicted value than those farther away. Thus, IDW assumes that each measured point has a local influence that diminishes with distance, hence the name 'inverse distance weighted'.

The general formula of IDW is:

$$
\widehat{D}\left(\lambda_{0}, \varphi_{0}\right)=\sum_{i=1}^{N} w_{i} D\left(\lambda_{i}, \varphi_{i}\right)
$$

where $\hat{D}\left(\lambda_{0}, \varphi_{0}\right)$ is the interpolated tropospheric delay for a location point with easting $\lambda_{0}$ and northing $\varphi_{0}, N$ is the number of GPS stations surrounding the prediction location that will be used in the interpolation, and $w_{i}(i=$ $1,2, \ldots N)$ are the weights assigned to each GPS-derived delay value that will be used. For IDW these weights will decrease with distance to the interpolated location. $D\left(\lambda_{i}, \varphi_{i}\right)$ is the GPS-derived delay (either singledifferenced or double-differenced) at location easting $\lambda_{i}$ and northing $\varphi_{i}$.

The weights are determined as follows: 


$$
w_{i}=\frac{d_{i 0}^{-p}}{\sum_{i=1}^{N} d_{i 0}^{-p}} \text { and } \sum_{i=1}^{N} w_{i}=1
$$

From equation (7) it can be seen that as the distance becomes larger, the weight is reduced by a factor of $p$. The quantity $\mathrm{d}_{\mathrm{i} 0}$ is the distance between the prediction location $\left(\lambda_{0}, \varphi_{0}\right)$ and each of the GPS stations $\left(\lambda_{i}, \varphi_{i}\right)$. The power parameter $p$ influences the weighting of the GPS-derived delay on the interpolated value: as the distance increases between the GPS stations and the prediction location, the weight (or influence) that the measured point will have on the prediction will decrease exponentially. By defining a high power, more emphasis is placed on the nearest points, and the resulting surface will have more detail (be less smooth). Specifying a lower power will give more influence to the points that are further away, resulting in a smoother surface. A power of 2 is most commonly used. The weights for the GPS-measured locations that will be used in the prediction are scaled so that their sum is equal to 1 .

\subsection{Spline interpolation}

This general-purpose interpolation method fits a minimum-curvature surface through the input points (Schultz 1973). Conceptually, this is like bending a sheet of rubber to pass through the points while minimizing the total curvature of the surface. It fits a mathematical function (a minimum-curvature, two-dimensional, thinplate spline) to a specified number of the nearest input points while passing through all input points. Therefore, the idea behind a spline fit is to approximate a function by a polynomial which is defined piecewise. This method is best for gradually varying surfaces. It is not appropriate when there are large changes within a short horizontal distance because it can overshoot estimated values. Hence, it would not be applicable to correct atmospheric interference induced by extreme weather conditions that may be caused by a cold front moving across the area.

For simplicity, the 1D 'basis' splines (B-splines) are described here, which became popular when de Boor (1978) developed a package of FORTRAN routines for their numerical application. For example, a cubic spline fit uses cubic polynomials which are defined over distinct, non-overlapping regions. The term spline means that the coefficients of the polynomial are chosen so that the following conditions are satisfied at the borders when two regions abut: (a) the values of the fit polynomials are the same, and (b) one or more of the derivatives match as well so that the slope (first derivative), etc., are continuous. For cubic splines, it is possible to match the function values and first derivatives (slopes) at both ends of the interval, resulting in a sufficiently smooth join for most purposes.

The idea behind B-splines is to expand the function in 'basis' splines $B(x)$, which are zero over most of the domain to be fitted. The $B(x)$ are splines, not simple polynomials - i.e. they are different polynomials in different regions. Consider the simplest useful B-splines, the cubic splines. The $B(x)$ will be non-zero in the region between $\mathrm{x}[\mathrm{i}]$ and $\mathrm{x}[\mathrm{i}+3]$, whereas $B(x)=0$ for $\mathrm{x}<\mathrm{x}[\mathrm{i}]$ or $\mathrm{x}>\mathrm{x}[\mathrm{i}+3]$. In order to be continuous, $B(x[i])=B(x[i+3])=0$. The function $B(x)$ can be written for a cubic B-spline as:

$$
\begin{aligned}
B(x)= & A(x-x[i])_{+}^{3}+B(x-x[i+1])_{+}^{3} \\
& +C(x-x[i+2])_{+}^{3}+D(x-x[i+3])_{+}^{3}
\end{aligned}
$$

where $(x-y)_{+}$is $(x-y)$ if $(x-y)>0$ and 0 otherwise. Thus, in the left-most interval, only the term proportional to A contributes, while in the right-most interval all of the terms contribute. The additional conditions on the derivatives of $B(x)$ at the end of the right-most interval, namely $B^{\prime}(x[i+3])=B^{\prime \prime}(x[i+3])=0$, result in the B-spline being unique up to a normalizing constant which multiplies B. The resultant B-splines are bell-shaped functions which are non-negative. B-splines can be defined for higher degrees but cubic B-splines are generally used in practice.

Beyond the endpoints of the domain there are points which are needed to define the B-spline at the edge of the domain. De Boor (1978) typically chooses $x[-3]=x[-2]=x[-1]=x[0]$ at the left-hand end, $x[0]$ being the endpoint of the domain, and similarly at the right-hand side. These points $x[i]$, at which the fit is defined, are typically called the knots or breakpoints of the splines. Often, the knot spacing is uniform within the domain, i.e. $\mathrm{x}[\mathrm{i}+1]-\mathrm{x}[\mathrm{i}]=\mathrm{dx}$ is a constant, although this is not necessary.

The process of fitting a function by splines involves determining the coefficients of the splines which satisfy the user-imposed conditions, which are typically to match the specified function and its derivatives on a set of points. This is done by building a spline approximation to the function from overlapping B-splines. The first cubic B-spline might cover the region from $\mathrm{x}[0]$ to $\mathrm{x}$ [3]; the next would be defined over $\mathrm{x}$ [1] to $\mathrm{x}$ [4], and so on. Recall that each cubic B-spline, as defined above, has one free parameter, its scale factor. Performing the fit requires determining this scale factor. Because at the ends of its range the B-spline takes on the value 0 , there will be three non-zero B-splines contributing to the value of the sum at each interior point. The linear system which must then be solved to fit the B-spline approximation to a set of function values is, then, a tri-diagonal system within the interior of the domain. Because of the multiple knots at the edges it is somewhat more 
complicated at either end. Such a system is still banded, and so generally can be solved without the complexity of a full system solver.

One interesting feature of B-splines is the locality of influence. The value of a function to be fitted influences only the coefficients of the B-splines which are non-zero over that interval. Thus, for cubic splines, only four coefficients are affected.

\subsection{Kriging interpolation}

This interpolation method assumes that the distance or direction between sample points reflects a spatial correlation that can be used to explain variations in the surface. Kriging fits a mathematical function to a specified number of points, or all points within a specified radius, to determine the output value for each location. Kriging is a multistep process including exploratory statistical analysis of the data, variogram modelling, creating the surface, and (optionally) exploring a variance surface (Stein 1999). This function is most appropriate when there is a spatially correlated distance or directional bias in the data.

Like IDW, kriging weights the surrounding GPS-measured values to derive a prediction for a non-measured location. The general formula for the kriging interpolator is the same as IDW, i.e. equation (6). However, in IDW the weight $w_{i}$ depends solely on the distance to the prediction location. Kriging also takes into account the overall spatial arrangement among the measured points by quantifying the spatial autocorrelation. Thus, in ordinary kriging, the weight $w_{i}$ depends on a fitted model to the measured points, the distance to the prediction location, and the spatial relationships among the measured values around the prediction location.

In order to create the empirical semivariogram the distance and squared difference between each pair of locations has to be calculated. The distance $d_{i j}$ between two locations $\left(\lambda_{i}, \varphi_{i}\right)$ and $\left(\lambda_{j}, \varphi_{j}\right)$ is determined by the

Euclidean distance:

$$
d_{i j}=\sqrt{\left(\lambda_{i}-\lambda_{j}\right)^{2}+\left(\varphi_{i}-\varphi_{j}\right)^{2}}
$$

The empirical semivariance $s_{i j}$ is half the square of difference between the GPS-derived tropospheric delay for the two locations:

$$
s_{i j}=\frac{1}{2}\left[D\left(\lambda_{i}, \varphi_{i}\right)-D\left(\lambda_{j}, \varphi_{j}\right)\right]^{2}
$$

With larger datasets (more GPS stations) the number of pairs of locations will increase rapidly and will quickly become unmanageable. Therefore it is necessary to group the pairs of locations, in a process referred to as 'binning'. In this case, a bin is a specified range of distances. That is, all points that are $0<d_{i j} \leq 1$ kilometre apart are grouped into the first bin, those that are $1<d_{i j} \leq 2$ kilometres apart are grouped into the second bin, and so forth. The average empirical semivariance of all pairs of points in a bin is taken as the semivariance of the bin.

Now the average semivariance can be plotted against the average distance of the bins, to produce the empirical semivariogram. However, the empirical semivariogram values cannot be used directly because standard errors for the predictions might be negative; instead, a model must be fitted to the empirical semivariogram. Once this is done, the fitted model can be used to determine semivariogram values for various distances. For simplicity, the model to be fitted is a least squares regression line, which has been forced to have a positive slope and pass through zero. Many other models can also be used. The slope $k$ of the regression line is then used to determine the semivariance $\gamma_{i j}$ at any given distance:

$$
\gamma_{i j}=k d_{i j}
$$

where $d_{i j}$ is the distance between two GPS stations at $\left(\lambda_{i}, \varphi_{i}\right)$ and $\left(\lambda_{j}, \varphi_{j}\right)$ calculated using equation (9). In order to interpolate the tropospheric delay at location $\left(\lambda_{0}, \varphi_{0}\right)$, a matrix $\Gamma$ and a vector $g$ can be defined using the semivariance of equation (11):

$$
\Gamma=\left[\begin{array}{cccc}
\gamma_{11} & \ldots & \gamma_{1 N} & 1 \\
\vdots & \ddots & \vdots & \vdots \\
\gamma_{N 1} & \ldots & \gamma_{N N} & 1 \\
1 & \ldots & 1 & 0
\end{array}\right], \quad g=\left[\begin{array}{c}
\gamma_{01} \\
\vdots \\
\gamma_{0 N} \\
1
\end{array}\right]
$$

The $1 \mathrm{~s}$ and 0 s in the bottom row and the right-most column of $\Gamma$, as well as the last element in $\boldsymbol{g}$, arise due to unbiasedness constraints. Now that the matrix $\Gamma$ and the vector $\boldsymbol{g}$ have been defined, the kriging weights vector $\boldsymbol{w}$ can be solved for:

$$
w=\left[\begin{array}{c}
w_{1} \\
\vdots \\
w_{N} \\
m
\end{array}\right]=\Gamma^{-1} g
$$


where $\Gamma^{-1}$ is the inverse matrix of $\Gamma$. The $m$ is an unknown to be estimated, arising from unbiasedness constraints.

Therefore, the interpolation can now be carried out using equation (6). It should be noted that kriging uses the GPS-derived delay data twice: the first time to estimate the spatial autocorrelation of the data, and the second to make the predictions.

\section{Experimental data analysis: SCIGN}

Data from the Southern California Integrated GPS Network (SCIGN 2003) were used to investigate the feasibility of the above methods to derive tropospheric delay corrections from GPS observations. Of the 23 stations considered, 14 were treated as measured locations (reference stations) and nine were used as prediction locations for which tropospheric delay corrections had to be determined and compared with their GPS-derived delays. A 2-hour session was observed on August 2, 2001 (DOY 214) and again on September 6, 2001 (DOY 249), simulating a typical ERS SAR satellite single repeat cycle of 35 days. Data were collected at a 30 s sampling rate for a period of one hour before and after the flyover of the radar satellite. Figure 1 shows the location of the GPS sites within a typical ERS SAR image frame (the dashed lines) for this area. A close-up of the GPS sites is also shown, where the reference stations are denoted by triangles, while the sites to be interpolated are indicated by circles.
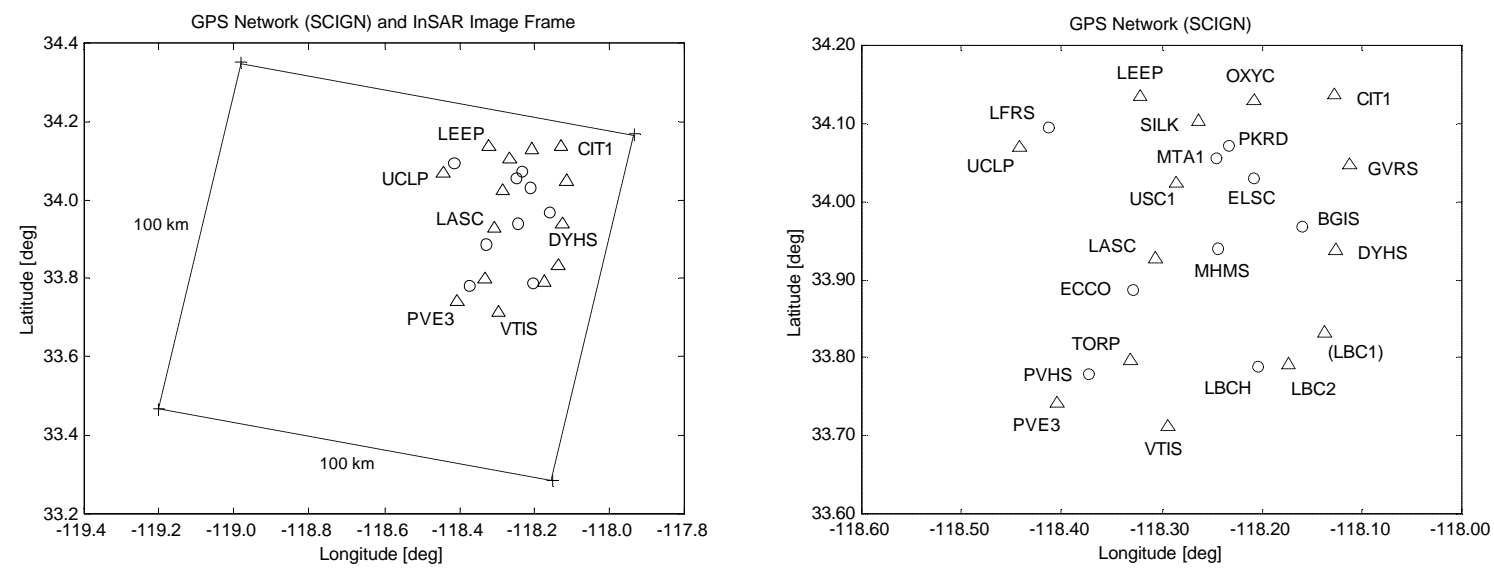

Figure 1. SCIGN stations within the ERS SAR image frame (left), and a close-up showing reference stations (triangles) and prediction stations (circles) (right)

For all sites precise coordinates were obtained using the Scripps Coordinate Update Tool (SCOUT) provided by the Scripps Orbit and Permanent Array Center (SOPAC 2003). This service computes the coordinates of a GPS receiver (whose data are submitted to the website) by using the three closest SCIGN reference sites and precise GPS ephemerides. In this case the coordinates were determined by taking the mean of six 24-hour solutions obtained in two blocks of three successive days (DOY 213-215 and 248-250). The average baseline lengths ranged from $2-7 \mathrm{~km}$. The repeatability of these six coordinate solutions was at the sub-centimetre level for all but one GPS site, indicating a solid, stable network. Site LBC1 showed relatively large coordinate variations indicating lower quality data or a possible displacement of $3.5 \mathrm{~cm}$ and has therefore been left out of the subsequent interpolation.

\subsection{GPS-derived tropospheric delay corrections}

The Bernese GPS processing software (Rothacher and Mervart 1996) was used to process the network on both days, the coordinates of CIT1 being held fixed as the primary reference station. Baseline lengths vary from $7 \mathrm{~km}$ to $49 \mathrm{~km}$, and the largest height difference is $270 \mathrm{~m}$. For each site tropospheric delay corrections were determined every 20 minutes, resulting in six parameters per site throughout the 2-hour observation span. Singledifferenced tropospheric corrections (equation 3) were then obtained by forming the differences relative to CIT1. These corrections range from $-6.1 \mathrm{~cm}$ to $+2.2 \mathrm{~cm}$, and in some cases show variations of a few centimetres within the 2-hour observation span.

Radar interferometry applications use two images of the same area in order to detect any ground deformation that might have occurred between the two satellite flyovers. To correct such an InSAR image for the effect of the tropospheric delay, the relative change in the tropospheric conditions is of great importance. Hence doubledifferenced tropospheric corrections are obtained by forming the between-epoch difference of the singledifferenced values derived in the previous step (equation 5). A comparison of the single- and double-differenced corrections revealed that almost all the double-differenced delay is smaller than the single-differenced delay (except for stations OXYC, MTA1 and PKRD). The double-differenced corrections range from $-5.0 \mathrm{~cm}$ to $+3.3 \mathrm{~cm}$ although the 23 stations spread over only a quarter of the SAR image frame (figure 1). Therefore, it is 
crucial to apply such corrections in order for InSAR to achieve sub-centimetre accuracy.

\subsection{Interpolation of tropospheric delay corrections}

For each of the nine prediction sites shown in figure 1, the tropospheric delay corrections were interpolated using the three methods described earlier: inverse distance weighted (IDW) interpolation, spline interpolation and kriging interpolation. Both the single-differenced tropospheric corrections relative to CIT1 for days 214 and 249, and the double-differenced tropospheric corrections between these two epochs were investigated by comparing the interpolated values to the 'true' values obtained directly using the Bernese software. This was done for each of the six 20-minute time intervals (Delay 1 through to Delay 6) within the 2-hour observation span.

Figures 2-4 show the interpolation images obtained for the different interpolation methods in the doubledifferenced case, which is most important and can be directly used for the correction of InSAR results. The dots indicate the locations of the 22 GPS stations used in the analysis (refer to figure 1 for their codes) and the colour/grey step interval is $1 \mathrm{~mm}$. The main areas of tropospheric activity can be recognised in all three figures, and the temporal and spatial variability of the tropospheric delay is obvious. The double-differenced interpolation values obtained with the different interpolation methods only differ by small amounts and are generally below or just above the cm-level. However, they $d o$ reach values of up to $3 \mathrm{~cm}$ in some cases.

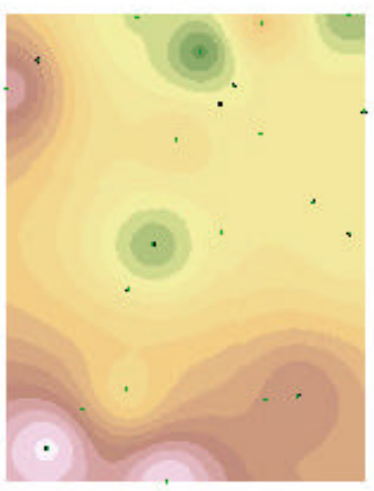

Delay 1

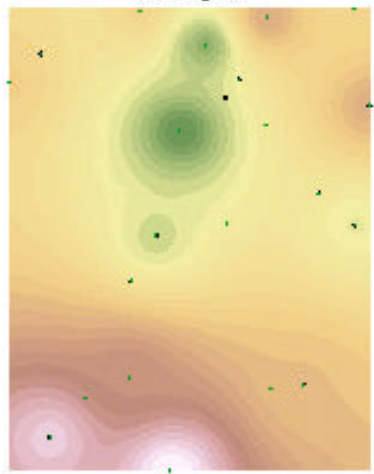

Delay 4

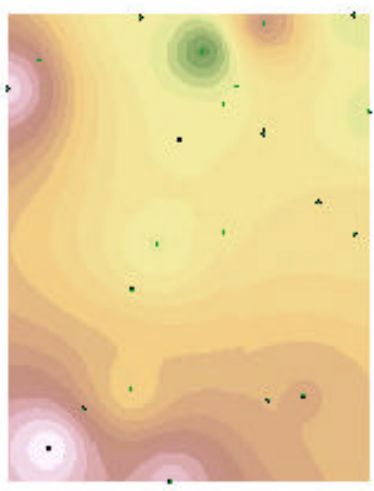

Delay 2

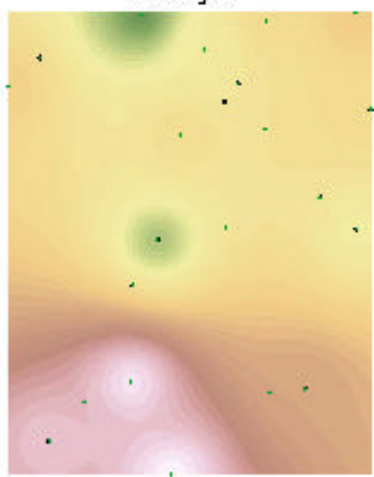

Delay 5

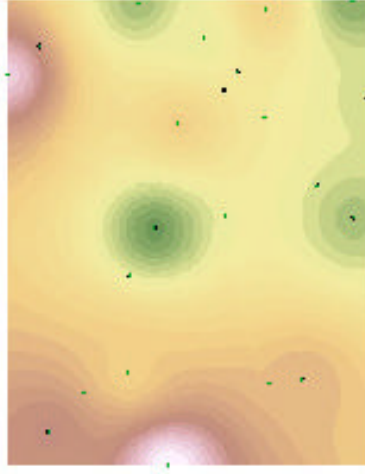

Delay 3

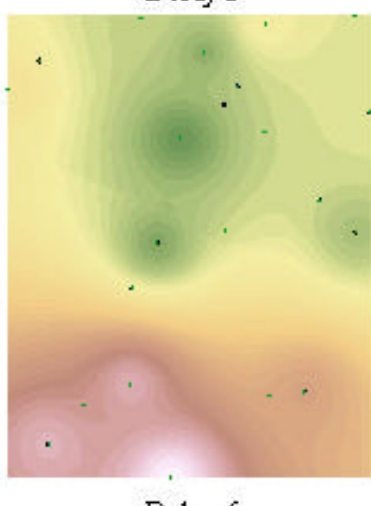

Delay 6

Figure 2. Interpolation images for double-differenced tropospheric corrections (IDW) 


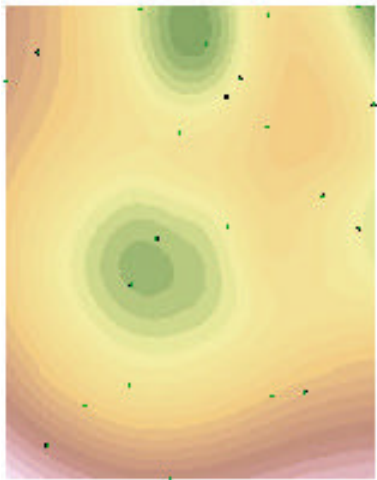

Delay 1

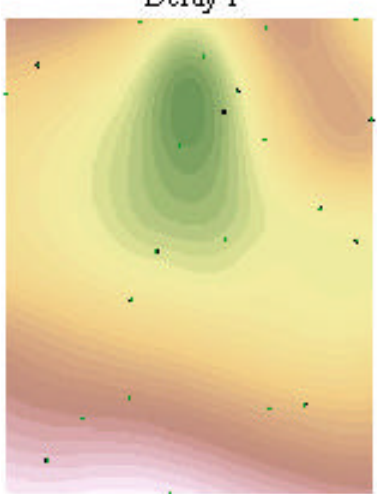

Delay 4

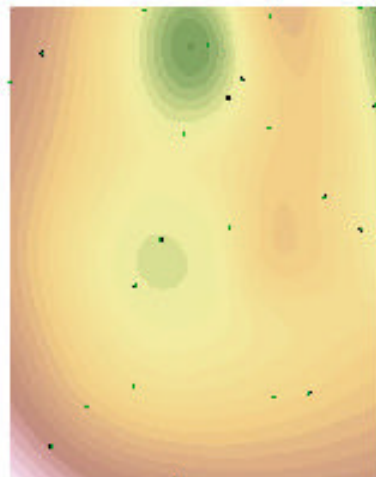

Delay 2

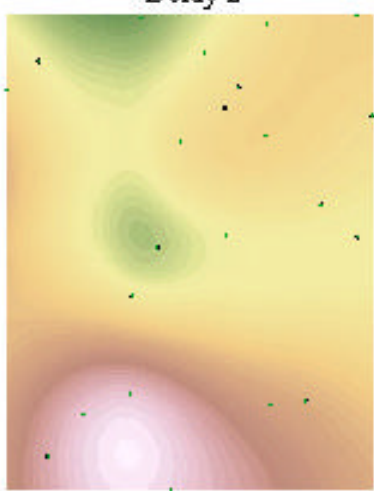

Delay 5

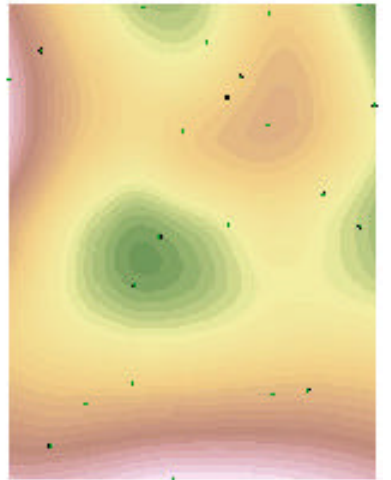

Delay 3

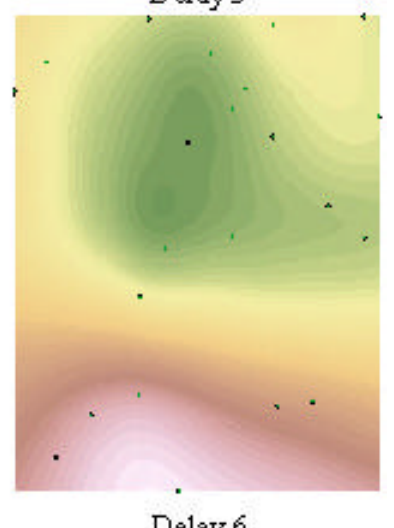

Delay 6

Figure 3. Interpolation images for double-differenced tropospheric corrections (Spline)
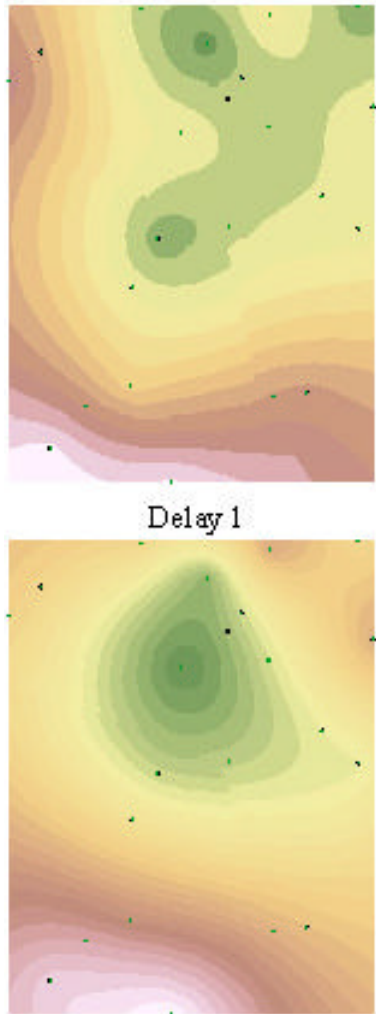

Delay 4

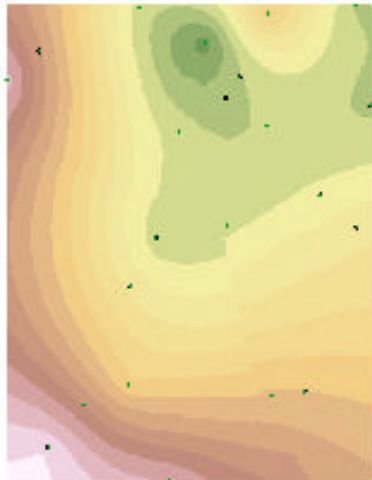

Delay 2

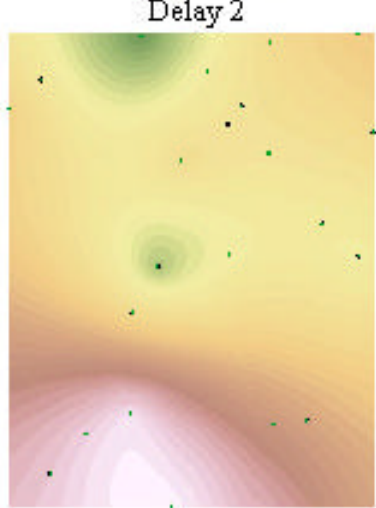

Delay 5

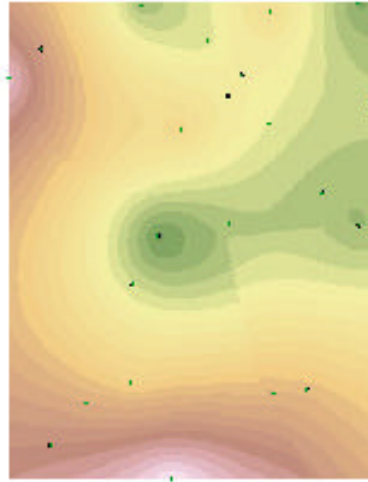

Delay 3

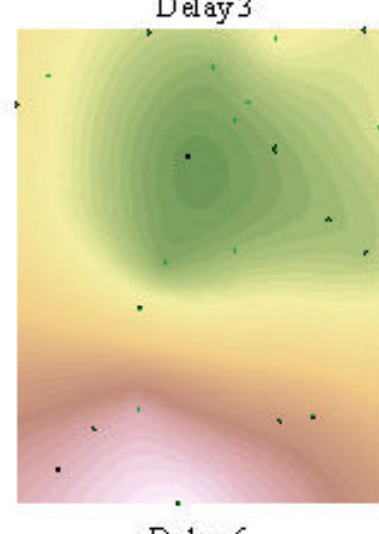

Delay 6

Figure 4. Interpolation images for double-differenced tropospheric corrections (Kriging) 


\subsection{Which interpolation method is the most suitable?}

In order to determine which interpolation method gives the best results, the standard deviations of the results compared to the 'true' values obtained using the Bernese software were computed. The left graph of figure 5 shows the standard deviations for the single-differenced case on days 214 (top plot) and 249 (middle plot), as well as for the double-differenced case (bottom plot). It is obvious that all three interpolation techniques deliver results with the same accuracy in this particular case, which is mostly at the sub-centimetre level. For the fourth time interval the accuracy is considerably lower compared to the rest of the observation span, almost reaching the $2 \mathrm{~cm}$ level. This may have been caused by a short-term tropospheric event on day 249 , which again highlights the importance of applying the differential tropospheric delay corrections to InSAR results.

The tropospheric delay corrections are to be used to correct a set of InSAR images obtained from two SAR satellite flyovers. Hence it is important that the reference stations (GPS-measured locations) do not undergo any deformation between these two epochs. In practice, however, small movements may still occur. These can be due to minor tectonic events, nearby construction work, or if the GPS site is in fact just inside the deformation zone to be monitored. It is therefore useful to test the susceptibility of the interpolation techniques to outliers caused by small displacements in the reference stations or by reduced data quality. LBC1, a site that had earlier been identified as having a problem, was now included as a reference station in the interpolation process. The data were then processed again. The standard deviations of the resulting tropospheric corrections for the singledifferenced case on days 214 (top plot) and 249 (middle plot), as well as for the double-differenced case (bottom plot), are shown in the right graph of figure 5.
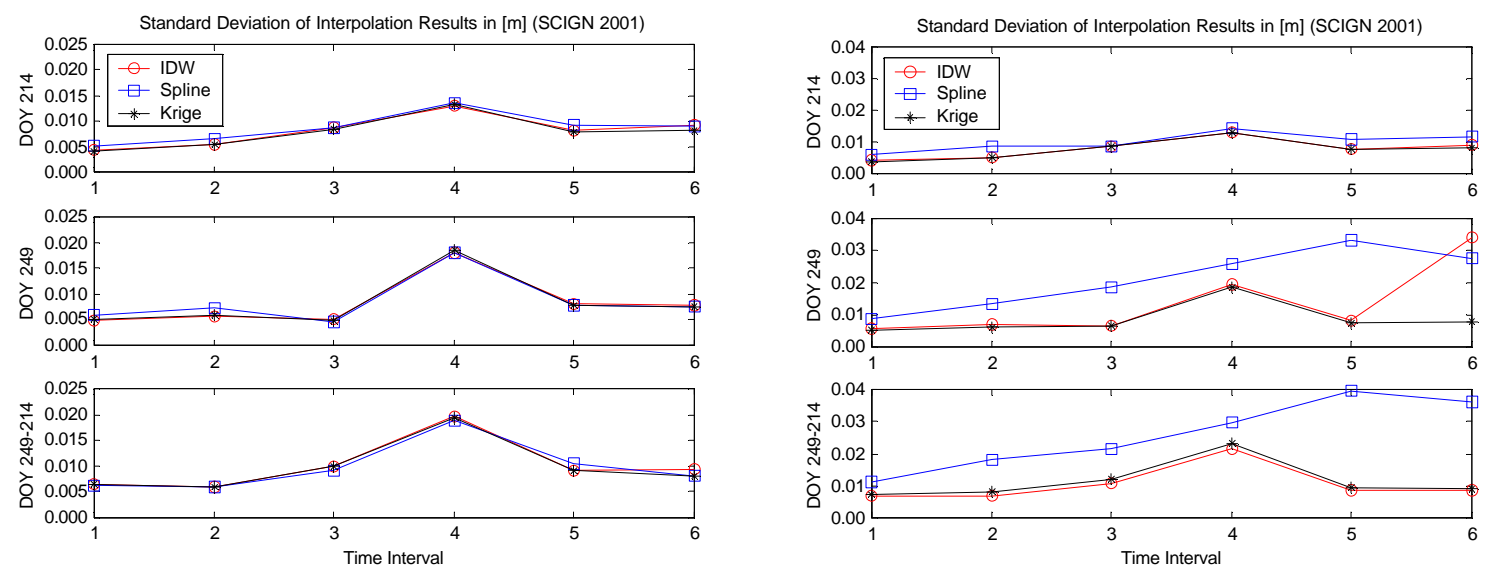

Figure 5. Standard deviation of the interpolation results obtained by different methods for a 'clean' reference network (left) and including an 'outlier' (right)

It is obvious that the spline interpolation method has difficulties coping with such an 'outlier' in the reference station network. Standard deviations reach values of up to $4 \mathrm{~cm}$ in the double-differenced case. The values for the IDW and kriging interpolation techniques remain unchanged compared to the 'clean' reference network used in the previous case. Only the sixth time interval of the IDW interpolation on day 249 shows a change for the worse. However, this does not influence the double-differenced result (bottom right graph of figure 5), which indicates the robustness of the method. It is therefore suggested that either the IDW or the kriging interpolation method be used to determine tropospheric delay parameters from GPS observations. On the other hand, the two techniques can be used as a mutual check.

\subsection{How many troposphere parameters should be determined?}

The Bernese GPS processing software allows the user to specify the number of tropospheric delay parameters to be determined. The estimation of about 6-12 parameters for a 24-hour observation session is recommended by Rothacher and Mervart (1996). Estimating one parameter for every 2-4 hours may be sufficient for geodetic control surveys where a set of coordinates is derived from a long observation session, taking into account all possible atmospheric effects. However, a special situation arises when one is dealing with GPS-derived tropospheric corrections for InSAR. The SAR satellite will pass over the area of interest at a certain epoch and we are specifically interested in estimating the tropospheric delay as accurately as possible at this epoch within the observation span. It is therefore necessary to determine how many parameters should be estimated in order to obtain an accurate representation of the tropospheric conditions at any point in time.

A sub-network involving three GPS sites from the original network (figure 1) was used. The baselines CIT1UCLP and CIT1-VTIS are $30 \mathrm{~km}$ and $49 \mathrm{~km}$ in length with height differences of $104 \mathrm{~m}$ and $156 \mathrm{~m}$ respectively. The 2-hour session observed on September 6, 2001 (DOY 249) was processed several times incorporating a different number of estimable troposphere parameters. Tropospheric delay corrections were estimated for time 
intervals of 20,10, 5 and 3 minutes in length, corresponding to 6, 12, 24 and 40 parameters per site respectively. Figure 6 shows the (single-differenced) tropospheric delay parameters for the sites UCLP (top) and VTIS (bottom), both relative to CIT1.

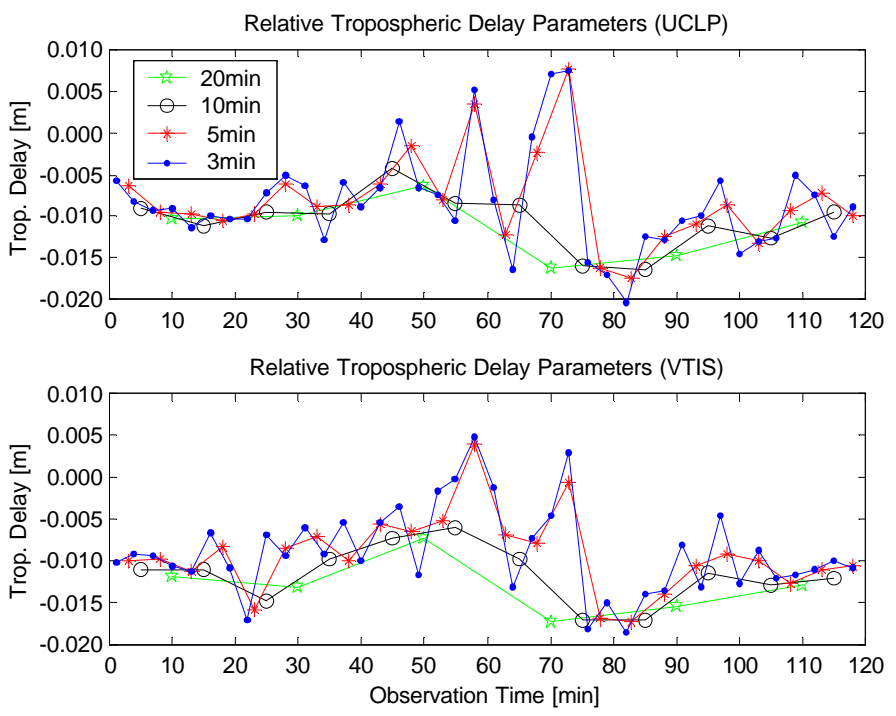

Figure 6. Relative tropospheric delay parameters over 2 hours

The figure indicates that both the $3 \mathrm{~min}$ and $5 \mathrm{~min}$ cases generate a rather detailed record of the variations in the troposphere. Short-term fluctuations are visible and values range from about $+1 \mathrm{~cm}$ to $-2 \mathrm{~cm}$, even for the relatively small height differences of $100-150 \mathrm{~m}$ between the stations. The $10 \mathrm{~min}$ and $20 \mathrm{~min}$ cases produce a smoothed record of the tropospheric delay, which is obviously less likely to represent the correct conditions present at a specific SAR time epoch. The resulting coordinates are practically the same for both the $3 \mathrm{~min}$ and $5 \mathrm{~min}$ tropospheric parameter estimation, with variations at the sub-mm level. If compared to the results obtained using 10min and 20min intervals, the coordinate differences are at the few-mm level. This corresponds to a difference of a few millimetres in the troposphere parameters between the $3 \mathrm{~min}$ and $5 \mathrm{~min}$ cases on the one hand and the $10 \mathrm{~min}$ and $20 \mathrm{~min}$ cases on the other (figure 6).

It should be noted, however, that the short-term fluctuations could also in part represent noise. Independent data are needed in order to distinguish noise from the signal. This is currently under investigation.

These results suggest that by estimating tropospheric delay parameters for 5-minute time intervals during a 2hour observation session, the short-term variations of the troposphere can be reliably modelled. At the same time the number of additional parameters to be estimated is still kept at a reasonable level.

\section{Experimental data analysis: GEONET}

Based on the above findings a second dataset from Japan's GPS Earth Observation Network (GEONET) (GSI 2003) was analysed. Of the 37 stations considered, 29 were treated as measured locations (reference stations) and eight were used as prediction locations for which tropospheric delay corrections had to be determined and compared with their GPS-derived delays. A 2-hour session was observed on June 17, 2002 (DOY 168) and on July 22, 2002 (DOY 203), again simulating a typical ERS SAR satellite single repeat cycle of 35 days, and covering the satellite flyover epoch. Figure 7 shows the location of the GPS sites, evenly distributed across a typical ERS SAR image frame (the dashed lines) for this area. The reference stations are denoted by triangles, while the sites to be interpolated are indicated by circles. Precise coordinates for all sites were provided by the Geographical Survey Institute (GSI) of Japan. 


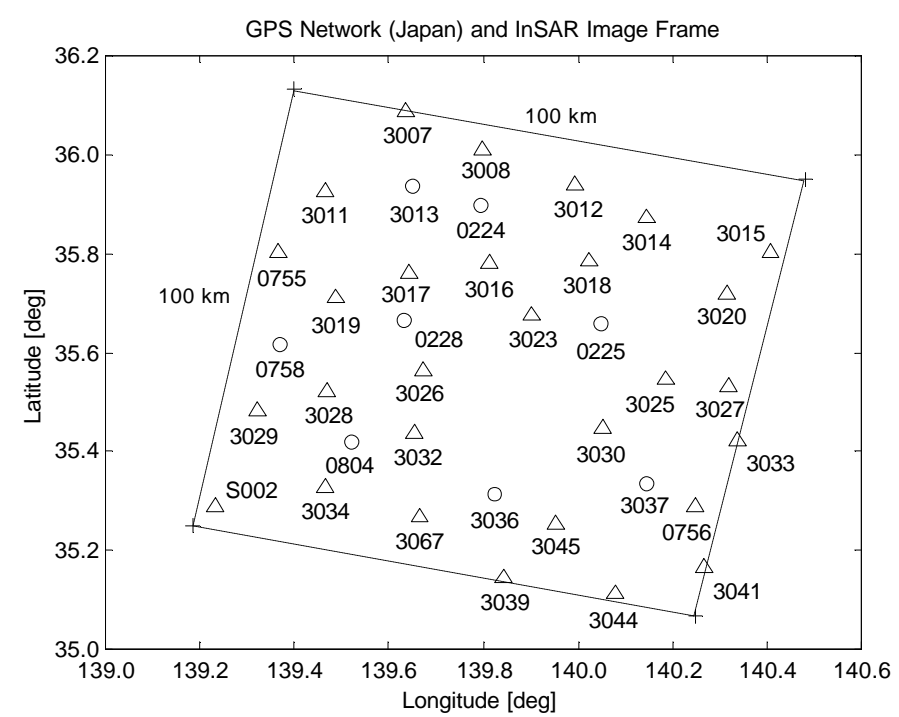

Figure 7. GEONET stations within the ERS SAR image frame

6.1 GPS-derived tropospheric delay corrections

Again, the Bernese GPS processing software was used to process the network on both days, the coordinates of S002 being held fixed as the primary reference station. Baseline lengths vary from $22 \mathrm{~km}$ to $121 \mathrm{~km}$, and the largest height difference is $321 \mathrm{~m}$. For each site tropospheric delay corrections were determined every 5 minutes, resulting in 24 parameters per site throughout the 2-hour observation span. It should be noted that in practice the primary reference station should be situated in, or close to, the centre of the SAR image frame in order to keep the baseline lengths to a minimum. In this analysis, however, the results obtained over longer baselines are also of interest.

Single-differenced tropospheric corrections (equation 3) were determined by forming the differences relative to S002. These corrections range from $-9.5 \mathrm{~cm}$ to $+4.2 \mathrm{~cm}$, showing variations of up to a few centimetres within the 2-hour observation span. Double-differenced tropospheric delay corrections were then obtained by forming the between-epoch difference of the single-differenced values derived in the previous step (equation 5). The double-differenced corrections range from $-6.7 \mathrm{~cm}$ to $+10.9 \mathrm{~cm}$, indicating significant changes in the tropospheric conditions (see also figure 8).

\subsection{Interpolation of tropospheric delay corrections}

For each of the eight prediction sites shown in figure 7, the tropospheric delay corrections were interpolated using the inverse distance weighted (IDW) interpolation method. Both the single-differenced tropospheric corrections relative to S002 for days 168 and 203, and the double-differenced tropospheric corrections between these two epochs were investigated by comparing the interpolated values to the 'true' values obtained directly using the Bernese software. This was done for each of the 24 -minute time intervals within the 2-hour observation span.

As an example, figure 8 shows two 3D interpolation 'maps' obtained in the double-differenced case, for the $11^{\text {th }}$ and $24^{\text {th }}$ time interval ( 65 minutes apart). The spatial and temporal variability of the troposphere can easily be recognised. Similar 'maps' can be generated for the single-differenced case and distributed as a routine CGPS product, with minimum effort, to aid radar interferometry.

Figure 9 shows the double-differenced corrections for the eight prediction sites, obtained for each of the 24 time intervals. The graphs show the parameters determined by the Bernese software, the interpolated values using the IDW method, and the differences between the two. It can be seen that the interpolation results agree very well with the 'true' values. The standard deviations of the differences are all (with one exception) at the sub-centimetre level, even for baselines of $85 \mathrm{~km}$ in length (table 1). 

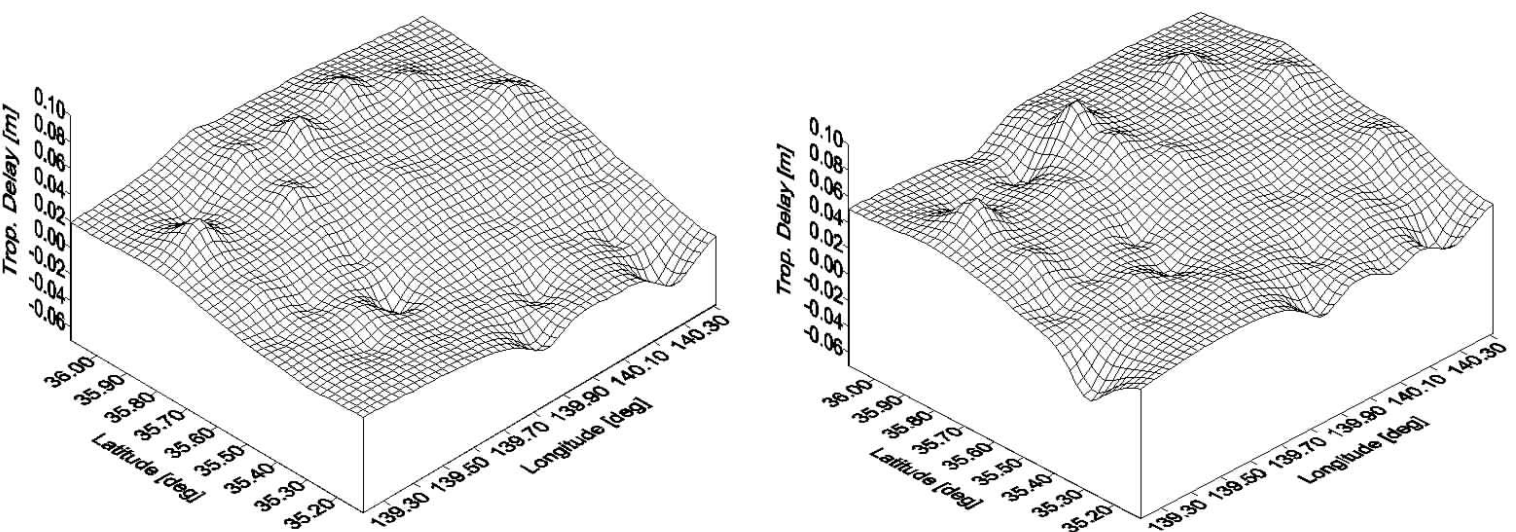

Figure 8. Double-differenced interpolation maps for the $11^{\text {th }}$ (left) and the $24^{\text {th }}$ (right) time interval (IDW interpolation)
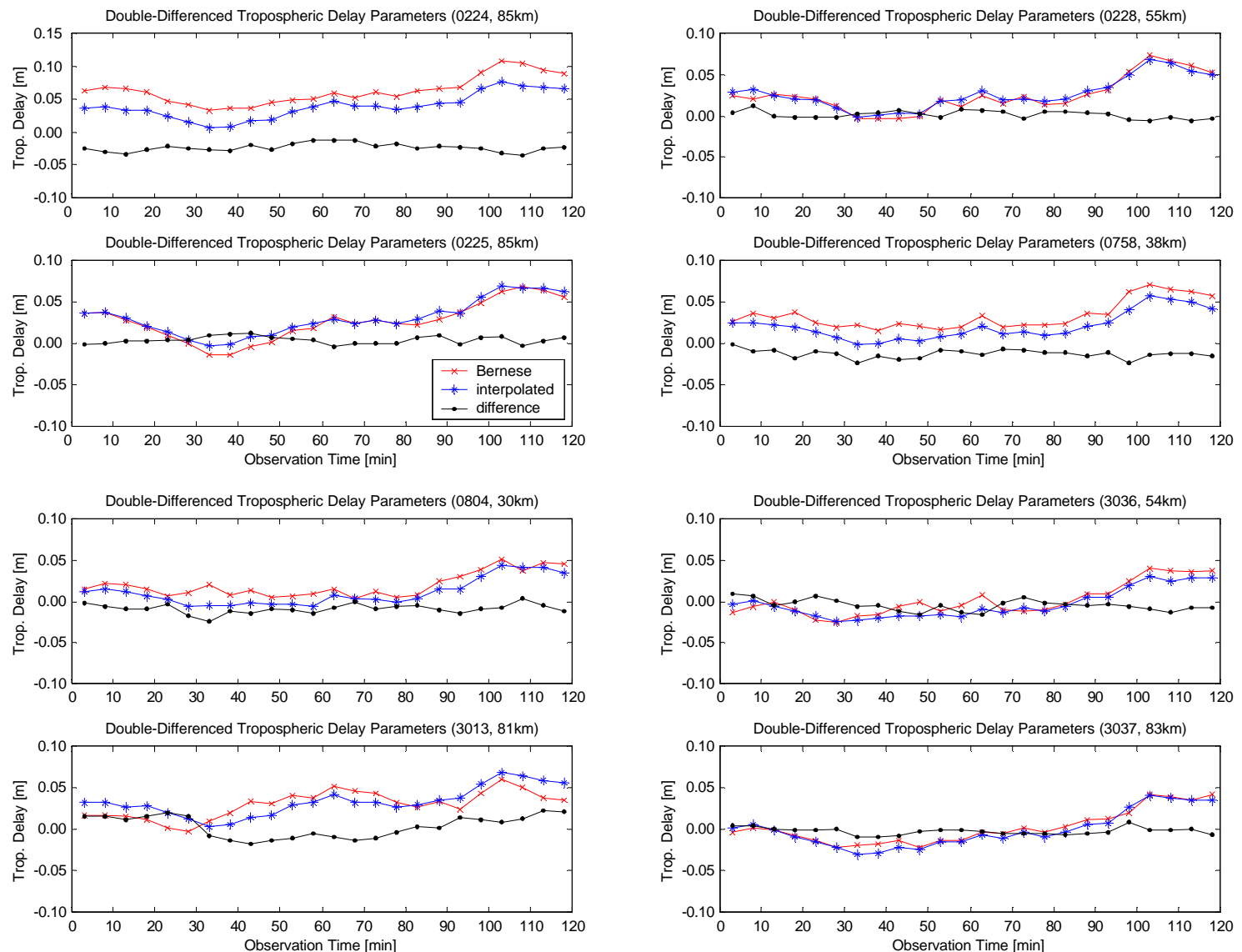

Figure 9. Comparison of Benese-derived and interpolated double-differenced tropospheric corrections

Table 1. Standard deviations of the differences between Bernese-derived and interpolated troposphere corrections

\begin{tabular}{ccc}
\hline Site & STD $[\mathrm{m}]$ & Baseline length $[\mathrm{km}]$ \\
\hline 0224 & 0.00625 & 85 \\
0225 & 0.00445 & 85 \\
0228 & 0.00472 & 55 \\
0758 & 0.00510 & 38 \\
0804 & 0.00597 & 30 \\
3013 & 0.01323 & 81 \\
3036 & 0.00697 & 54 \\
3037 & 0.00450 & 83 \\
\hline
\end{tabular}




\section{Conclusions}

Tropospheric heterogeneity (differential tropospheric delay) can lead to misinterpretation of InSAR results. A between-site and between-epoch double-differencing algorithm to derive tropospheric corrections to radar results from GPS observations has been tested. These GPS measurements can be collected by either a network of continuous GPS (CGPS) stations or GPS campaigns synchronised to the radar satellite flyover. In order to correct the radar result on a pixel-by-pixel basis, the GPS-derived corrections have to be interpolated. Three interpolation methods, namely the inverse distance weighted, spline, and kriging techniques, have been investigated. Using GPS data from two test networks, it has been found that the inverse distance weighted and kriging interpolation methods are more suitable. Differential corrections as much as several centimetres may have to be applied in order to ensure sub-centimetre accuracy for the radar result. It seems optimal to estimate the tropospheric delay from GPS data at 5-minute intervals.

How many GPS sites are actually required to achieve a given level of accuracy for the corrections depends on the tropospheric conditions present in the area. This includes the geographic location, the extend of the area under investigation, and the height differences between the GPS network sites. These factors determine the maximum distance between the GPS stations that would still allow the troposphere to be adequately modelled. Obviously the reference sites should be evenly distributed across the area to maximise the quality of the interpolation results.

The algorithm and procedures described in this paper could easily be implemented in a CGPS network data centre. The interpolated grid of between-site, single-differenced tropospheric delays can be generated as a routine product to assist radar interferometry, in a manner similar to the SLC radar images.

\section{Acknowledgements}

SCIGN and its sponsors, the W.M. Keck Foundation, NASA, NSF, USGS and SCEC, as well as Japan's Geographical Survey Institute are acknowledged for providing the GPS data used in this study. Mr Yufei Wang is thanked for his assistance with the interpolation algorithms.

\section{References}

Bauersima I (1983) NAVSTAR/Global Positioning System (GPS) II: Radiointerferometrische Satellitenbeobachtungen. Mitteilungen der Satellitenbeobachtungsstation Zimmerwald, Berne, Switzerland, vol. 10

Black HD (1978) An easily implemented algorithm for the tropospheric range correction. Journal of Geophysical Research 83(B4): 1825-1828

Bock Y, Williams S (1997) Integrated satellite interferometry in Southern California. Eos 78(29): 293

De Boor C (1978) A practical guide to splines. Springer, New York

Essen L, Froome KD (1951) The refractive indices and dielectric constants of air and its principal constituents at $24000 \mathrm{mc} / \mathrm{s}$. Proc. of Physical Society 64(B): 862-875

Ge L, Ishikawa Y, Fujiwara S, Miyazaki S, Qiao X, Li X, Yuan X, Chen W, Wang J (1997) The integration of InSAR and CGPS: A solution to efficient deformation monitoring. Proc. Int. Symp. on Current Crustal Movement \& Hazard Reduction in East Asia and South-East Asia, 4-7 November 1997, Wuhan, P. R. China, $145-155$

Ge L (2000) Development and testing of augmentations of continuously-operating GPS networks to improve their spatial and temporal resolution. UNISURV S-63, The University of New South Wales, Australia

Graham LC (1974) Synthetic interferometer radar for topographic mapping. Proc. IEEE 62(6): 763-768

GSI (2003) http://mekira.gsi.go.jp/ENGLISH/index.html

Hanssen RF (2001) Radar interferometry - Data interpretation and error analysis. Kluwer Academic Publishers, Dordrecht-Boston-London

Hanssen RF, Weckwerth TM, Zebker HA, Klees R (1999) High-resolution water vapor mapping from interferometric radar measurements. Science 283: 1295-1297

Hopfield HS (1969) Two-quartic tropospheric refractivity profile for correcting satellite data. Journal of Geophysical Research 74(18): 4487-4499

Lancaster P, Salkauskas K (1986) Curve and surface fitting: An introduction. Academic Press, London-Orlando Lu Z, Fatland R, Wyss M, Li S, Eichelberger J, Dean K, Freymueller J (1997) Deformation of New Trident volcano measured by ERS-1 SAR interferometry, Katmai National Park, Alaska. Geophysical Research Letters 24(6): 695-698

Massonnet D, Rossi M, Carmona C, Adragna F, Peltzer G, Feigl K, Rabaute T (1993) The displacement field of the Landers earthquake mapped by radar interferometry. Nature 364: 138-142

Mendes VB (1999) Modeling the neutral-atmosphere propagation delay in radiometric space techniques. Dept. of Geodesy \& Geomatics Eng. Tech. Rept. No. 199, University of New Brunswick, Fredericton, Canada 
Rothacher M, Mervart L (Eds) (1996) Bernese GPS Software Version 4.0. Astronomical Institute, University of Berne, Switzerland

Saastamoinen J (1973) Contributions to the theory of atmospheric refraction. Bulletin Géodésique 107: 13-34

Schultz MH (1973) Spline analysis. Prentice-Hall, Englewood Cliffs, N.J.

SCIGN (2003) http://www.scign.org/

SOPAC (2003) http://sopac.ucsd.edu/cgi-bin/SCOUT.cgi

Spilker JJ (1996) Tropospheric effects on GPS. In Global Positioning System: Theory and applications I, vol. 163, edited by Parkinson BW and Spilker JJ, American Institute of Aeronautics and Astronautics, Washington, 517-546

Stein ML (1999) Interpolation of spatial data: Some theory for kriging. Springer, New York

Zebker HA, Rosen PA, Hensley S (1997) Atmospheric effects in interferometric synthetic aperture radar surface deformation and topographic maps. Journal of Geophysical Research 102(B4): 7547-7563 